\title{
Spiky density of states in large complex Al-Mn phases
}

\author{
G. Trambly de Laissardière \\ Laboratoire de Physique Théorique et Modélisation, \\ CNRS and Université de Cergy-Pontoise, 2 av. A. Chauvin, F-95302, Cergy-Pontoise, France. \\ Email: guy.trambly@u-cergy.fr
}

\begin{abstract}
First-principle electronic structure calculations have been performed in crystalline complex phases $\mu-\mathrm{Al}_{4.12} \mathrm{Mn}$ and $\lambda-\mathrm{Al}_{4.6} \mathrm{Mn}$ using the TB-LMTO method. These atomic structures, related to quasicrystalline structures, contain about 560 atoms in a large hexagonal unit cell. One of the main characteristic of their density of states is the presence of fine peaks the so-called "spiky structure". From multiple-scattering calculations in real space, we show that these fine peaks are not artifacts in ab-initio calculations, since they result from a specific localization of electrons by atomic clusters of different length scales.
\end{abstract}

\section{INTRODUCTION}

Al based Quasicrystals, approximants and related complex metallic phases reveal very unusual transport properties (for review see Ref. [1] and Refs. therein). Indeed, they exhibit a very high resistivity although the density of states (DOS) at the Fermi energy $E_{\mathrm{F}}$ is not very small. This means that their transport properties are due mainly to a small diffusivity of electrons. Recently [2], we proposed a new mechanism for the conductivity in those systems where the velocity of charge carriers is very low. This mechanism is related with a specific localization of electrons by complex atomic structures. Several numerical studies argue that this localization leads to fine peaks in the DOS, called "spiky peaks". Thus, spiky DOS were predicted in icosahedral small approximants (for instance $\alpha \mathrm{Al}-\mathrm{Mn}-\mathrm{Si}$ 1/1-approximant [3, 4], 1/1 Al$\mathrm{Cu}-\mathrm{Fe}$ [5], 1/1 Al-Pd-Mn [6]). In the case of crystalline approximants, it is obvious that these peaks are associated to a small electron velocity (flat dispersion relations) [3]. Such properties are not specific of quasicrystals as they are also observed in many crystal related to quasicrystals, therefore it does not come from the long range quasiperiodicity. Indeed, we have predicted [7] that fine peaks in the DOS could come from electron confinement by atomic clusters characteristic of the quasiperiodicity. This is not in contradiction with a Hume-Rothery mechanism for stabilizing these phases because this tendency to localization has a small effect on the band energy [7]. The existence of spiky DOS in quasicrystals and approximants with large unit cell is however still much debated experimentally (Refs. [8, 9, 10] and references therein) and theoretically (Refs. [7, 11, 12] and references therein). Very recently, R. Widmer et al. [10], found a clear signature of spiky local DOS near Fermi level in scanning tunneling microscopy and spectroscopy at low temperature.

In this paper, we present ab-initio calculsations and multiple scattering calculations that prove the existence on fine peaks in the DOS of complex Al-Mn crystals with large unit cell.

\section{FIRST-PRINCIPLE STUDY}

The $\mu-\mathrm{Al}_{4.12} \mathrm{Mn}$ phase is crystallized in a hexagonal structure $\left(\mathrm{P} 6_{3} / \mathrm{mmc}\right.$ space group) with large unit cell: $a=19.98(1) \AA$ and $c=24.673(4) \AA[13]$. Each unit cell contains $110 \mathrm{Mn}$ atoms and $\sim 453 \mathrm{Al}$ atoms. As explained in Ref. [14], we have modified slightly the Wyckoff position of one $\mathrm{Al}$ atom in order to avoid too short interatomic distances. The $\lambda-\mathrm{Al}_{4.6} \mathrm{Mn}$ crystalizes also in $\mathrm{P} 6_{3} / \mathrm{mmc}$ with $a=23.382(9) \AA$ and $c=12.389(2) \AA$ 15]. Its atomic arrangements have many similarities with those of $\mu-\mathrm{Al}_{4.12} \mathrm{Mn}$.

DOSs are calculated by using the self-consistent tight-binding linear muffin tin orbital (TB-LMTO) method [16], in the atomic sphere approximation (ASA) without spin polarization. The LMTO basis includes all angular momenta up to $l=2$. The Brillouin zone (BZ) integration on the $k$ space was done by the tetrahedron method with $N_{k} k$-points in a BZ. LMTO DOS of $\mu$ $\mathrm{Al}_{4.12} \mathrm{Mn}$ have been already calculated by D. NguyenManh and G. Trambly de Laissardière 14] for analyzing the origin of magnetism in complex aluminides related to quasicrystals [17, 18]. Here, we focus on the shape of the DOS without spin-polarization. To increase the accuracy of calculation, we increase the number $N_{k}$. In Ref. 14] TB-LMTO calculation in $\mu$ phase was performed with $N_{k}=9$, whereas $N_{k}=3375$ in actual calculation for $\mu$ phase, and $N_{k}=1458$, for $\lambda$ phase.

The total DOSs in $\mu$ and $\lambda$ phases are very similar (figures 1 and 21). At low energies, the parabola due to the $\mathrm{Al}$ nearly-free states is clearly seen. The large $d$ band (figure 2(b)) from -2 up to $1 \mathrm{eV}$ is due to a strong $s p$ $d$ hybridization [3, 6, 19, 20]. The sum of local DOSs on $\mathrm{Al}$ atoms, shown in figure 2(c), is mainly sp DOS. As expected for a Hume-Rothery stabilization, there is a wide pseudogap in $s p$ DOS near $E_{\mathrm{F}}[3,5,6,18,19,20]$. The pseudogap in the total DOS is narrower because the $d$ states of Mn atoms must fill up it partially [19]. As shown on the insert figure 1 DOS around $E_{\mathrm{F}}$ consists in a set of spiky peaks. Nevertheless the resolution is not well define in the TB-LMTO calculations [4], therefore these calculations are not enough to prove the existence of spiky peaks. 


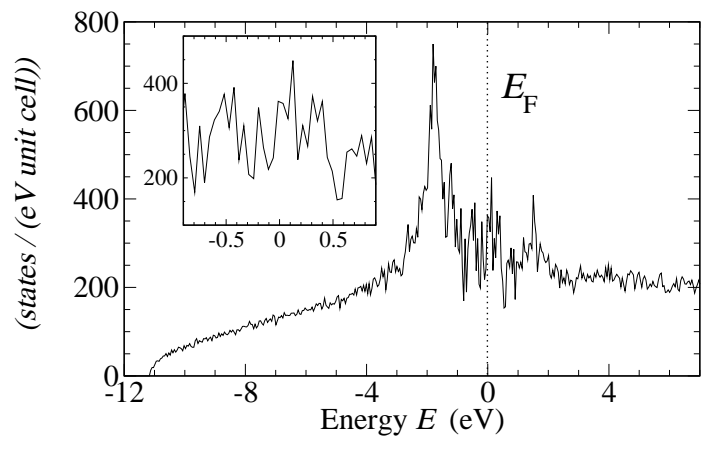

FIG. 1: Total LMTO DOS in $\mu$ - $\mathrm{Al}_{4.12} \mathrm{Mn}$. Insert: Total DOS around $E=0$.
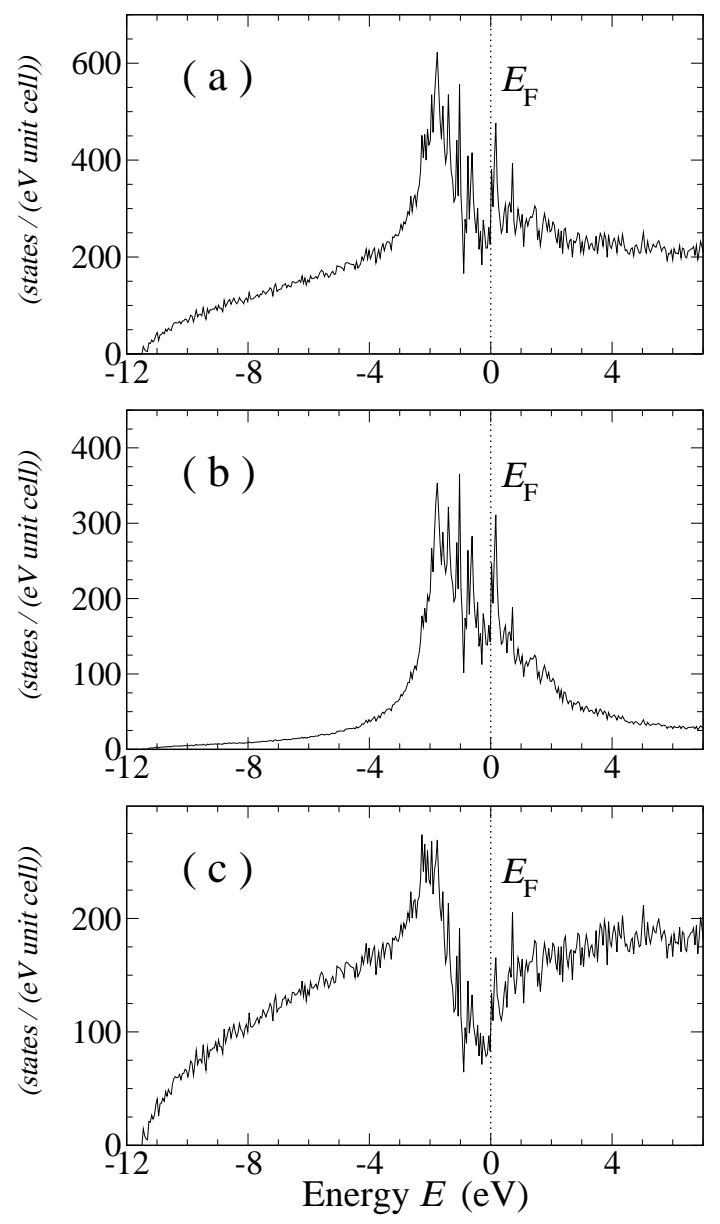

FIG. 2: LMTO DOS in $\lambda-\mathrm{Al}_{4.6} \mathrm{Mn}$ : (a) Total DOS, (b) local Mn DOS and (c) local Al DOS.

\section{SPIKY DENSITY OF STATES ?}

To prove the existence or not of fine peaks in DOS, we calculate the DOS for a large assembly of atoms by using a multiple scattering approach in real space. Therefore there is no error due to Brillouin zone (BZ) integration as with TB-LMTO method. Multiple scattering calculation

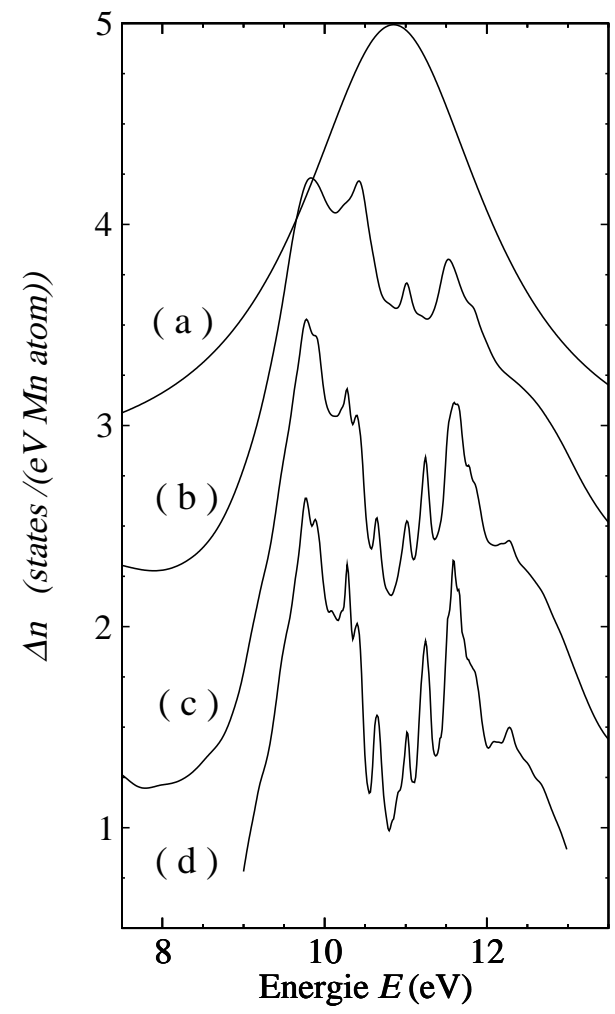

FIG. 3: Variation $\Delta n(E)$ of the total DOS due to $X \mathrm{Mn}$ atoms in free electron matrix. Mn atoms are located on the Mn sub-lattice of $\mu$ - $\mathrm{Al}_{4.12} \mathrm{Mn}$. (a) $X=1$ is the Virtual Bound States. (b) $X=110$ corresponds to 1 unit cell of $\mu$ phase. (c) $X=880,8$ unit cells of $\mu$ phase. (d) $X=1980,18$ unit cells of $\mu$ phase.

is not an ab-initio calculation, but it allows to discuss qualitatively the existence of spiky peaks.

In a standard description of intermetallic alloys, one starts from a muffin-tin potential, which varies in spheres centered on the atoms and is constant in the space between the spheres. As far as the band structure is concerned an atom is entirely characterized by its scattering properties for incident plane waves. The effect of a group of atoms on band structure will be characterized by its T-matrix, that is by its scattering properties of plane waves (Ref. 7] and Refs. therein). To simulate $\mathrm{Al}(\mathrm{Si})$ $\mathrm{Mn}$ phases, we consider only the potential due to the Mn atoms, which are strong scatterers, and we neglect the potential of all the $\mathrm{Al}$ (and $\mathrm{Si}$ ) atoms that are considered as weak scatterers. Furthermore, we consider that the Mn $d$ orbitals are the only source of scattering for the conduction electrons (i.e. the $s p$ electrons). Within these approximations a complex $\mathrm{Al}(\mathrm{Si})-\mathrm{Mn}$ phase becomes an assembly of $X$ atoms of $\mathrm{Mn}$ in a jellium simulating $\mathrm{Al}$ (Si) atoms (free $s p$ electron matrix). The total DOS of this system is:

$$
n(E)=n_{s p}^{\text {free }}(E)+\Delta n(E),
$$

where $n_{s p}^{f r e e}$ is the DOS of free electrons without $s p-d$ 


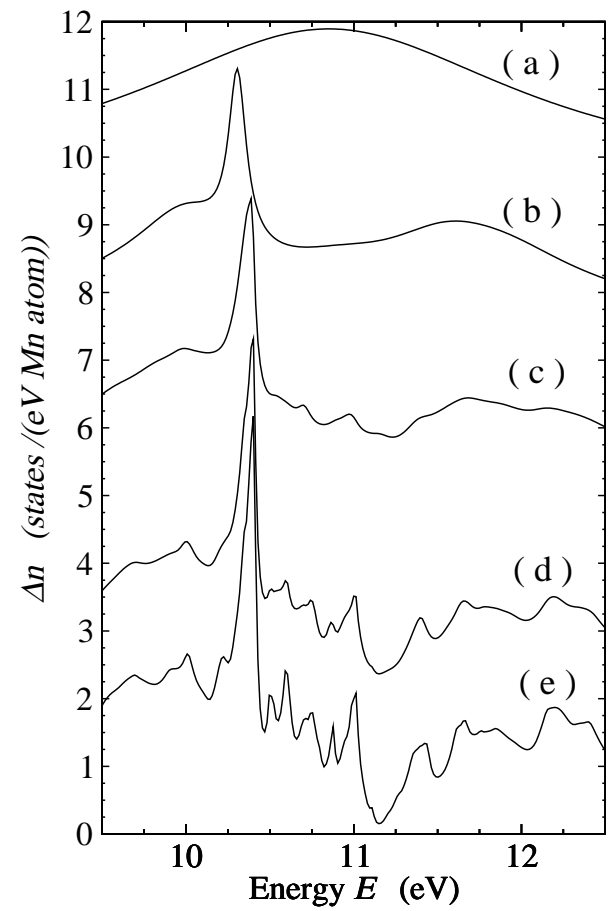

FIG. 4: Variation $\Delta n(E)$ of the total DOS due to $X$ Mn atoms in free electron matrix. Mn atoms are located on the Mn sublattice of $\alpha$-Al-Mn-Si cubic $1 / 1$ approximant. (a) $X=1$ is the Virtual Bound States. (b) $X=12,1 \mathrm{Mn}$ icosahedron. (c) $X=192,16 \mathrm{Mn}$ icosahedra on bcc lattice. (d) $X=648$, $54 \mathrm{Mn}$ icosahedra on bcc lattice. (e) $X=1536,128 \mathrm{Mn}$ icosahedra on bcc lattice.

interaction, and $\Delta n$, the variation of DOS due to the Mn atoms. $\Delta n(E)$ is calculated by using the Lloyd formula [7, 21], which is a generalization for several Mn atoms of the Friedel sum rule for one Mn atom in jellium:

$$
\Delta n(E)=-\frac{2}{\pi} \operatorname{Tr}\left(\mathbf{M}(E)^{-1} \frac{\mathrm{d}}{\mathrm{d} E} \mathbf{M}(E)\right) .
$$

The matrix elements of $\mathbf{M}(E)$ are calculated form the T-matrix:

$$
\mathbf{M}=\mathbf{t}^{-1}-\mathbf{G}
$$

where $\mathbf{G}$ is the propagator that coupled $d$ orbitals of different Mn scatters, and

$$
\mathbf{t}=\left(\begin{array}{lll}
t_{1} & & \\
& \ddots & \\
& & t_{X}
\end{array}\right) .
$$

$t_{x}$ is the element of the transfer matrix due to the $\mathrm{Mn}$ scatter indexed by $x . t_{x}$ depends only on the phase shift $\delta_{d}$ in wavefunction of the $s p$ states due to their scattering:

with

$$
\tan \delta_{d}(E)=\frac{-\Gamma}{E-E_{d}}
$$

$$
2 \Gamma=\pi V^{2} n_{s p}^{\text {free }}\left(E_{\mathrm{F}}\right)
$$

where $2 \Gamma$ is the width of the $d$ resonance and $E_{d}$ the on-site $d$ energy. $V$ is the coupling between one $d$ orbital and $s p$ eigenstates [19]. When $X=1$ (impurity limit), the total $s p$ DOS, $n_{s p}$, is not modified by the $s p$ - $d$ hybridization, and the variation $\Delta n$ of the total DOS due to the Mn atom is a Lorentzian (Virtual Bound State) centered on energy $E_{d}$ and with a width equals to $2 \Gamma$.

To simulate complex $\mathrm{Al}(\mathrm{Si})-\mathrm{Mn}$, the only parameters are the values of $E_{d}$ and $\Gamma$, and positions of Mn atoms in the real space. We use the following realistic values 19]: $E_{d}=10.9 \mathrm{eV}$ and $2 \Gamma=3 \mathrm{eV}$, and atomic positions determined from X-ray refinements. The DOS of several assemblies of $\mathrm{Mn}$ atoms are thus determined for $\mathrm{Mn}$ belonging to the Mn-sublattice of $\mu$-phase (figure 3) and $\alpha$-AlMnSi 1/1-approximant (figure 4). For $\mu$ and $\alpha$ structures, fine peaks appear in the DOS when a large number of Mn scatterers is taken into account (large value of $X$ ). Widths of these peaks are about $40-150 \mathrm{meV}$ which agrees with ab-initio calculations (figures 11 and 2 and Ref. 19]). Each fine peak is a signature of states confined by atomic clusters, so-called "Cluster Virtual Bound states" 7]. When the number of Mn scatters increases, new peaks appear. This means that atomic structure confines electrons on several length scales corresponding to different sizes of clusters, respectively.

Summarizing, multiple-scattering calculations have been performed in real space to simulate DOS of complex Al(rich)-Mn phases related to quasicrystals. They show that a localization of electrons by large assemblies of atoms leading to fine peaks in the DOS. This confirms the existence of the spiky DOS found by ab-initio calculations.

This work owes much to discusion with D. Mayou and D. Nguyen-Manh with whom ab-initio study of $\mu$-phase has been initiated. The computations have been performed at the Service Informatique Recherche (S.I.R.), Université de Cergy-Pontoise. I thank also Y. Costes and B. Marir, S.I.R., for computing assistance.
[1] Mayou, D.; Trambly de Laissardière, G.: Quantum transport in quasicrystals and complex metallic alloys. In Quasicrystals, series "Handbook of Metal Physics", (Eds T. Fujiwara, Y. Ishii), p. 209-265. Elsevier Science, 2008.
[2] Trambly de Laissardière, G.; Julien J. P.; Mayou, D.: Quantum Transport of Slow Charge Carriers in Quasicrystals and Correlated Systems. Phys. Rev. Lett. 97 (2006) 026601. 
[3] Fujiwara T.: Electronic structure in the Al-Mn alloy crystalline analog of quasicrystals. Phys. Rev. B40 (1989) 942-946. Fujiwara, T.; Yamamoto, S.; Trambly de Laissardière, G.: Band Structure Effects of Transport Properites in Icosahedral Quasicrystals. Phys. Rev. Lett. 71 (1993) 4166-4169.

[4] Zijlstra, E. S.; Bose S. K.: Detailed ab initio electronic structure study of two approximants to Al-Mn based icosahedral quasicrystals. Phys. Rev. B67, (2003) 224204.

[5] Trambly de Laissardière, G.; Fujiwara, T.: Electronic structure and conductivity in a model approximant of the icosaheral quasicrystals Al-Cu-Fe. Phys. Rev. B50 (1994) 5999-6005 .

[6] Krajčí, M., et al.: Atomic and electronic structure of icosahedral Al-Pd-Mn alloys and approximant phases. Phys. Rev. B 51 (1995) 17355-17378.

[7] Trambly de Laissardière, G.; Mayou, D.: Clusters and localization of electrons in quasicrystals Phys. Rev. B 55 (1997) 2890-2893. Trambly de Laissardière, G.; Roche, S.; Mayou, D.: Mat. Sci. Eng. A 226-228 986-989 (1997).

[8] Dolinšek J, et al.: Searching for sharp features in the pseudogap of icosahedral quasicrystals by NMR. Phys. Rev. B62 (2000) 8862-8870.

[9] Stadnik, Z. M., et al.: Absence of fine structure in the photoemission spectrum of the icosahedral Al-Pd-Mn quasicrystal. Phys. Rev. B64 (2001) 214202.

[10] Widmer, R., et al.: Low-temperature scanning tunneling spectroscopy on the 5-fold surface of the icosahedral AlPdMn quasicrystal. Phil.Mag. 86 (2006) 781-787. Maeder, R., et al.: private communication.

[11] Zijlstra, E. S.; Janssen, T.: Non-spiky density of states of an icosahedral quasicrystal Europhys. Lett. 52 (2000) 578-583.

[12] Trambly de Laissardière, G.: Interplay between electronic structure and medium range atomic order in hexagonal $\beta \mathrm{Al}_{9} \mathrm{Mn}_{3} \mathrm{Si}$ and $\varphi \mathrm{Al}_{10} \mathrm{Mn}_{3}$ crystals. Phys.
Rev. B 68 (2003) 045117.

[13] Shoemaker, C. B.; Keszler, D.; Shoemaker, D.: Structure of $\mu-\mathrm{MnAl}_{4}$ with Composition Close to that of Quasicrystal Phases. Acta Cryst. B45, (1989) 13-20.

[14] Nguyen-Manh D.; Trambly de Laissardière, G.: Firstprinciples predictions of magnetic properties for a complex and strongly related to quasicrystalline phase: $\mu$ $\mathrm{Al}_{4}$ Mn. J. Mag. Mag. Mater., 262 (2003) 496-501.

[15] Kreiner, G; Franzen, H.F.: The crystal structure of $\lambda$ $\mathrm{Al}_{4.6} \mathrm{Mn}$ J. Alloys Comp. 261 (1997) 83-104.

[16] Krier, G.; Jepsen, O.; Burkhart A.; Andersen, O. K.: The TB-LMTO program. Stuttgart, (1995).

[17] Simonet, V., et al.: Origin of magnetism in crystalline and quasicrystalline AlMn and AlPdMn phases. Phys. Rev. B58 (1998) R8865-R8868.

[18] Hippert, F., et al.: Magnetic properties of AlPdMn approximant phases J. Phys: Condens. Matter 11 (1999) 10419-10435. Trambly de Laissardière, G.; Mayou, D.: Magnetism in $\mathrm{Al}(\mathrm{Si})-\mathrm{Mn}$ quasicrystals and related phases Phys. Rev. Lett. 85 (2000) 3273-3276. Préjean, J. J.; Hippert, F.: 10th International Conference on Quasicrystals, Zurick, 2008.

[19] Trambly de Laissardière, G.; Nguyen-Manh D.; Mayou, D.: Electronic structure of complex Hume-Rothery phases and quasicrystals in transition metal aluminides. Prog. Mater. Sci. 50 (2005) 679-788.

[20] Z. Dankhazi, Z., et al.: Theoretical and experimental electronic distribution in $\mathrm{Al}_{6} \mathrm{Mn}$. J. Phys. Condens. Matter, 5 (1993) 3339-3350. Trambly de Laissardière, G., et al:: Experimental and theoretical electronic distributions in Al-Cu-based alloys. Phys. Rev. B51 (1995) 14035-14047. Belin, E; Mayou, D.: Electronic properties of quasi-crystals. Phys. Scr. T 49 (1993) 356-359.

[21] Gonis A.: Green Functions for Ordered and Disordered Systems. (Ets: E. van Groesen and E.M. de Jager) Studies in Mathematical Physics, vol. 4. North Holland, Amsterdam 1992. 